\title{
Introduction to "Scalable inference for Markov processes with intractable likelihoods" by J. Owen, D. Wilkinson, C. Gillespie
}

\author{
Robin J. Ryder
}

Published online: 30 October 2014

(C) Springer Science+Business Media New York 2014

Approximate Bayesian computation (ABC) was one of the major themes of MCMSki 2014, with several talks and especially many posters devoted to advances and applications of this method. The approach of Owen, Wilkinson and Gillespie is original, since it uses ABC not to perform direct (approximate) sampling from the posterior, but as an exploratory tool to be used to find a good initialization point for another technique.

For the area of application considered (dynamical systems), there as long been a debate about the most useful method between $\mathrm{ABC}$, which gives an approximate answer in a not-unreasonable time, and particle Markov chain Monte Carlo (pMCMC), which is asymptotically exact but easily runs into convergence issues. By using the output of $\mathrm{ABC}$ as an initialization for pMCMC, we can get the best of both worlds. This of course generalizes to other MCMC algorithms.

The authors have presented convincing results on the Lotka-Volterra model and other dynamical systems. These present the peculiarity that $\mathrm{ABC}$ is significantly faster than the MCMC burn-in, a condition for the approach to be useful. A further gain comes from the fact that $\mathrm{ABC}$ is much easier to parallelize than any MCMC scheme. Even when taking parallelization into account, this condition will not be typically met in practice, but the application to dynamical systems is certainly not the only one in which this trick will allow significant speed-ups. 